www.jmscr.igmpublication.org

Impact Factor 5.244

Index Copernicus Value: 83.27

ISSN (e)-2347-176x ISSN (p) 2455-0450

crossref DOI:_http://dx.doi.org/10.18535/jmscr/v4i7.53

Journal Of Medical Science And Clinical Research

\title{
Correlating the Severity of Chronic Kidney Disease with Oral Health: A Prospective Observational Study
}

\author{
Authors \\ Dr Mayilananthi K ${ }^{1}$, Dr Durga Krishnan², Dr Sarah Premraj ${ }^{3}$, Dr Bhaya Mathivanan ${ }^{4}$, \\ Prof. Dr Rajasekaran $\mathrm{D}^{\mathbf{5}}$ \\ ${ }^{1}$ Associate Professor, Department of General Medicine \\ ${ }^{2}$ Associate Professor, Department of General Medicine \\ ${ }^{3}$ Assistant Professor, Department of General Medicine \\ ${ }^{4}$ Chettinad Dental College \\ ${ }^{5} \mathrm{HOD}$ and Professor, Department of General Medicine
}

\begin{abstract}
Background: Chronic kidney disease (CKD) is defined as a progressive and most often, irreversible decline in renal function. Changes in the oral cavity, such as periodontitis, oral candidiasis, xerostomia, halitosis, dental caries and other manifestations, are common in patients with chronic kidney disease. Only a few studies have been done to establish the relationship between different oral manifestations in various stages of CKD. Hence this study was conducted to analyze the presence of oral diseases in adults with CKD and explore the association between the various oral manifestations in different stages of CKD.

Methods: 100 patients with Chronic Kidney Disease in various stages grouped by Glomerular Filtration Rate (GFR) were screened. 89 patients who met the selection criteria were enrolled in the study. After history and physical examination, detailed oral examination was done along with collection of saliva. The results were correlated with various stages of CKD.

Results: In our study, we have examined 89 patients who are having various stages of chronic kidney disease. On which, decay was present in 34\% of patients, xerostomia was present in 19\% of patients, periodontitis was present in $99.4 \%$ of patients which is highly significant, and oral candidiasis was present in $31 \%$ of patients.
\end{abstract}

Conclusion: There is an increased risk of periodontal disease in patients with CKD. There is an inverse relationship between the periodontal disease and GFR. Oral manifestations such as decay, oral candidiasis, and xerostomia are also noted with increased frequency in Chronic Kidney Disease patients.

Key words: Chronic Kidney Disease, oral manifestations, periodontitis.

\section{Introduction}

Chronic kidney disease (CKD) is a disorder affecting the structure and function of the kidneys. Chronic kidney disease can be diagnosed based on the presence of kidney damage (albuminuria) or decreased function of kidneys for 3 months or more $^{1}$. There is an increased prevalence of kidney disease in India in recent years and the number of patients diagnosed to have CKD have been increasing dramatically each year. It is estimated that nearly 229 per million populations have End Stage Renal disease and greater than 100,000 new patients enter renal replacement programs annually in India ${ }^{2}$. 
Chronic renal failure patients are more prone to have systemic infections which in turn will lead to inflammatory reactions ${ }^{3}$. This persistent inflammation gets reflected as various symptoms and signs in every organs of our body. Patients with CKD are having high risk of developing oral complications ${ }^{4}$. Oral health status of CKD and dialysis patients is poorer while comparing with healthy human beings and is often ignored.

Several studies showed that uremic patients have higher rates of decayed, missing teeth, loss of attachment, and mucosal lesions than the general population $^{(4)}$. The reasons postulated for these manifestations include persistent inflammatory response that is noted in $\mathrm{CKD}$, treatment for CKD (drugs, dialysis or transplantation), advancing age, common co-morbidities like diabetes, and a state of immune dysfunction that may increase the risk for periodontitis and other oral and dental pathologic conditions.

Chronic renal disease can give rise to a wide spectrum of oral manifestations affecting the hard or soft oral tissues ${ }^{(5)}$. Oral pathological manifestations include an ammonia-like odor resulting from high urea content, malocclusion, gingivitis, periodontitis, oral candidiasis, gingival hyperplasia, halitosis, salivary dysfunction, pallor of oral mucosa, clinical attachment loss, and dental mobility.

Periodontal disease is defined as a chronic inflammatory condition of the tissues surrounding the teeth. This is usually associated with accumulation of bacteria with resultant tooth loss (6). The causes for increased prevalence/severity of periodontal disease in CKD are not completely understood, but the possible mechanisms include diminished salivation and xerostomia, malnutrition, impaired immunity and wound healing, poor oral hygiene, alveolar bone destruction due to renal osteodystrophy, bleeding diathesis, and diabetes mellitus ${ }^{(7)}$. The serum level of osteocalcin and/or GCF osteocalcin in CKD patients are increased and this may also explain the effect of CKD on periodontal disease. It is noted that high inflammatory response seen in CKD contribute to the aggravated periodontal status, and it correlates well with the high level of C-reactive protein that increases over time in these patients ${ }^{(8)}$.

A main factor associated with oral candidiasis is a diminished host resistance as a result of CKD. Candidiasis may exhibit a variety of clinical patterns including pseudomembranous, erythematosus, median rhromboid glossitis, chronic multifocal, angular cheilitis, denture stomatitis, hyperplastic, mucocutaneous and endocrine-candidiasis syndromes. The fungal infection is more common in patients with CKD who suffer from hyposalivation and xerostomia.

\section{Aims and objectives}

The aim of this study was to find out the various oral manifestations in Chronic Kidney Disease patients and to find out its relationship with the severity (stages) of Chronic Kidney Disease.

\section{Materials and Methods}

The study was a prospective cross sectional study carried out at a semi urban tertiary care centre in South India, after obtaining necessary approval from the institutional ethics committee. Informed consent was obtained from all the participants prior to initiation into the study.

\section{Inclusion criteria}

- Based on classification of chronic kidney disease by National Kidney Foundation Kidney Disease Outcomes Quality Initiative of 2002, patients who had glomerular filtration rate below $89 \mathrm{ml} / \mathrm{min} /$ $1.73 \mathrm{~m} 2$ were included in this study. Glomerular filtration rate was calculated using Cockcroft-Gault formula ${ }^{(9)}$.

\section{Exclusion criteria}

- Patients with acute renal failure

- Patients who are receiving drugs that could affect the oral health status.

Demographic details of the CKD patients including name, age, sex, occupation, place and 
personal history, history and duration of any systemic illness were obtained. Examination of cardiovascular system, respiratory system, abdomen, foot examination was done to acquire the information about the normal physiological functions of the body.

Biochemical findings from their case records were utilized that include renal function test ( BUN by urease, creatine by jaffe's kinetic method, uric acid by uricase method ), sodium and potassium, urine complete, urine for microalbuminuria, and if the patient is diabetic fasting blood sugar and post prandial blood sugar by hexokinase method, Glycated haemoglobin by HbAlc(auto analyzer).

ECG for cardiac assessment and Ultrasonography of abdomen for kidney size estimation were also done.

\section{Grouping of patients}

Stage 1: Kidney damage with normal or increased GFR (>90 mL/min/1.73 m2)

Stage 2: Mild reduction in GFR (60-89 $\mathrm{mL} / \mathrm{min} / 1.73 \mathrm{~m} 2$ )

Stage 3: Moderate reduction in GFR (30-59 $\mathrm{mL} / \mathrm{min} / 1.73 \mathrm{~m} 2$ )

Stage 4: Severe reduction in GFR (15-29 $\mathrm{mL} / \mathrm{min} / 1.73 \mathrm{~m} 2$ )

Stage 5: Kidney failure (GFR $<15 \mathrm{~mL} / \mathrm{min} / 1.73$ $\mathrm{m} 2$ or dialysis)

\section{Oral examination and investigations}

The CPITN is an epidemiologic tool developed by the WHO for the evaluation of periodontal disease in population surveys ${ }^{(10)}$. It can be used to recommend the kind of treatment needed to prevent periodontal diseases according to standardized clinical criteria. The highest community periodontal index code was recorded in each segment

code 0: No signs of periodontal disease,

code 1: Gingival bleeding after gentle probing, code 2: Supragingival or subgingival calculus, code 3: 4-5 mm deep pathologic pockets, code 4: $6 \mathrm{~mm}$ or deeper pathologic pockets and code X: Missing index teeth

\section{Fungus examination}

0.5-ml amount of uncentrifuged saliva was spread on Sabouraud glucose agar plates containing 50 $\mathrm{mg}$ of chloramphenicol per $\mathrm{ml}$; plates were incubated at $37^{\circ} \mathrm{C}$ for $48 \mathrm{~h}$, and the number of CFU per milliliter of saliva was counted ${ }^{(11)}$. Assessment included evidence of clinical manifestations of candidiasis and a quantitative measure of Candida pseudohyphae in a cytologic smear from the midline posterior dorsal tongue ${ }^{(12)}$. The data was collected through a questionnaire and entered into contingency tables. Statistical analysis was carried out. The significance was determined using the Fisher's exact test and a ' $p$ ' value of less than 0.05 was considered statistically significant.

\section{Results}

100 patients were screened out of which 89 were recruited for the study after fulfilling the inclusion criteria. This study was conducted over a period of 2 months from July 2015 to August 2015. On examining 89 chronic renal failure patients, decay was present in $34 \%$ of patients, xerostomia was present in $19 \%$ of patients, periodontitis was present in $99.4 \%$ of patients which is highly significant, oral candidiasis was present in $31 \%$ of patients. The results were shown in figure.1

In our study on examining 89 patients, mild periodontitis was present in $6.7 \%$ of patients, moderate periodontitis was present in $32 \%$ of patients and severe periodontitis was present in $60.7 \%$ of patients. The results were represented in figure 2 .

Correlation of stages of CKD with various oral manifestations was studied and the results are represented in Table 1. There is a strong corelation existing between the periodontitis and GFR values. Patients who are having low GFR rate have higher prevalence of periodontitis and patients who are having high GFR are most likely have mild form of periodontitis. Hence, periodontitis was inversely proportional to GFR. 
Fig.1: Spectrum of oral manifestations in patients with CKD

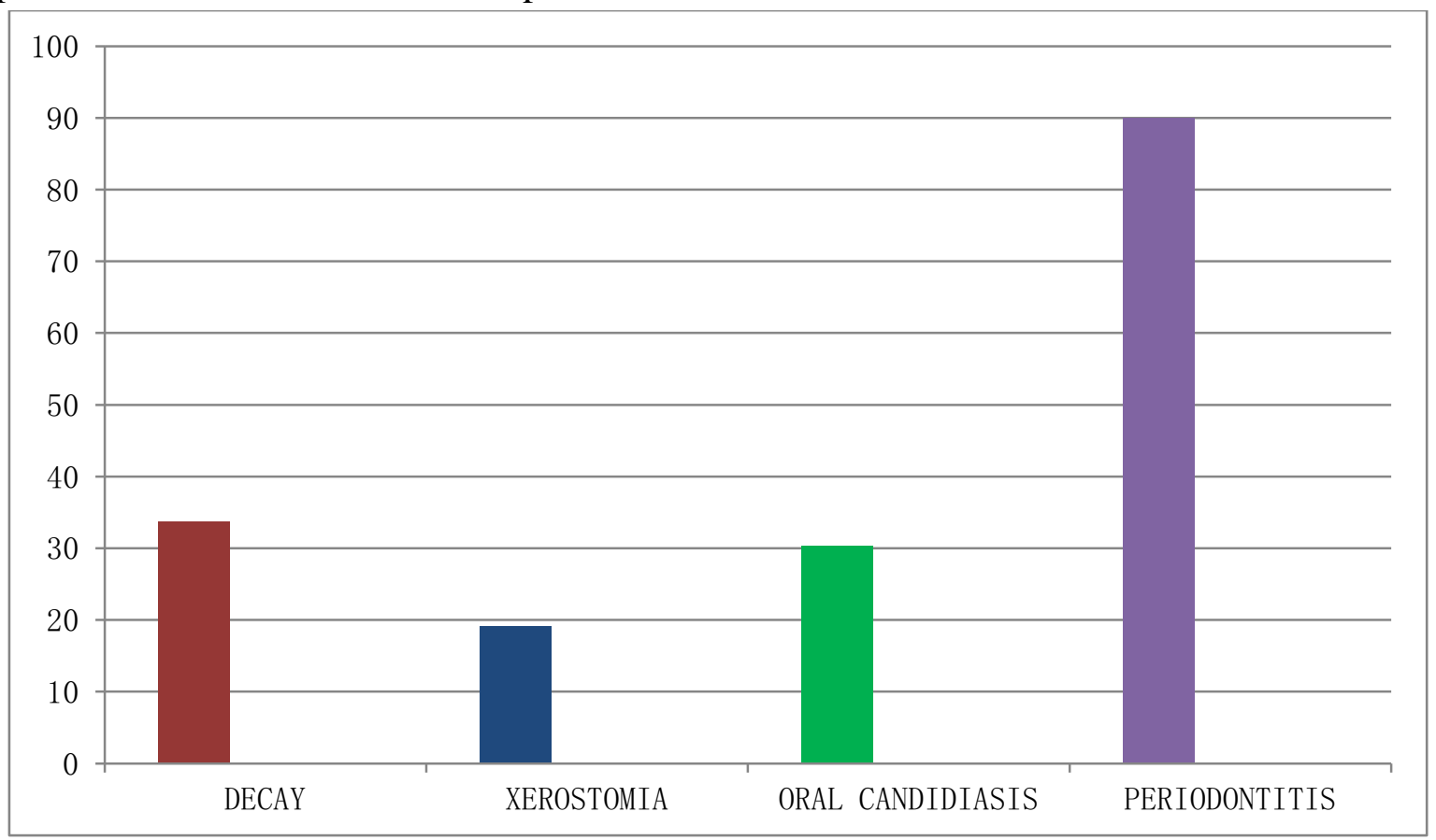

Fig.2: Degree of periodontitis in patients with CKD

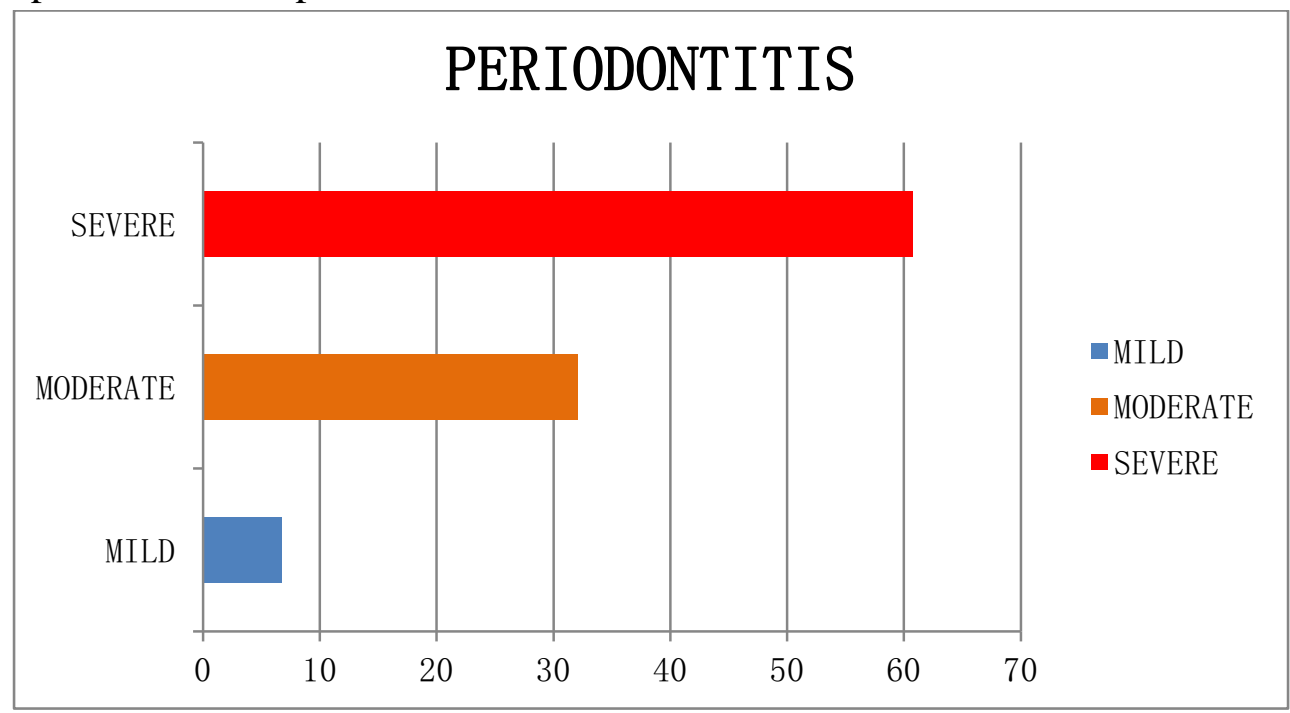


Tab.1: Correlative data of CKD staging and oral manifestations Correlations

\begin{tabular}{|c|c|c|c|c|c|c|c|c|}
\hline & & Weight & SCR & GFR & Decay & PI & XERO & $\mathrm{OC}$ \\
\hline \multirow[t]{3}{*}{ Weight } & Pearson Correlation & 1 & .042 & .186 & -.148 & -.078 & -.033 & .051 \\
\hline & Sig. (2-tailed) & & .694 & .082 & .165 & .470 & .758 & .637 \\
\hline & $\mathrm{N}$ & 89 & 89 & 89 & 89 & 89 & 89 & 89 \\
\hline \multirow[t]{3}{*}{ SCR } & Pearson Correlation & .042 & 1 & $-.769^{* * *}$ & -.151 & $.372^{* *}$ & .069 & .091 \\
\hline & Sig. (2-tailed) & .694 & & .000 & .158 & .000 & .520 & .398 \\
\hline & $\mathrm{N}$ & 89 & 89 & 89 & 89 & 89 & 89 & 89 \\
\hline \multirow[t]{3}{*}{ GFR } & Pearson Correlation & .186 & $-.769^{* * *}$ & 1 & .072 & $-.532^{* *}$ & -.129 & -.115 \\
\hline & Sig. (2-tailed) & .082 & .000 & & .503 & .000 & .228 & .282 \\
\hline & $\mathrm{N}$ & 89 & 89 & 89 & 89 & 89 & 89 & 89 \\
\hline \multirow[t]{3}{*}{ Decay } & Pearson Correlation & -.148 & -.151 & .072 & 1 & -.007 & $-.226^{*}$ & .046 \\
\hline & Sig. (2-tailed) & .165 & .158 & .503 & & .949 & .034 & .665 \\
\hline & $\mathrm{N}$ & 89 & 89 & 89 & 89 & 89 & 89 & 89 \\
\hline \multirow[t]{3}{*}{ PI } & Pearson Correlation & -.078 & $.372^{* *}$ & $-.532^{* *}$ & -.007 & 1 & .085 & $.254^{*}$ \\
\hline & Sig. (2-tailed) & .470 & .000 & .000 & .949 & & .431 & .016 \\
\hline & $\mathrm{N}$ & 89 & 89 & 89 & 89 & 89 & 89 & 89 \\
\hline \multirow[t]{3}{*}{ XERO } & Pearson Correlation & -.033 & .069 & -.129 & $-.226^{*}$ & .085 & 1 & -.134 \\
\hline & Sig. (2-tailed) & .758 & .520 & .228 & .034 & .431 & & .210 \\
\hline & $\mathrm{N}$ & 89 & 89 & 89 & 89 & 89 & 89 & 89 \\
\hline \multirow[t]{3}{*}{$\mathrm{OC}$} & Pearson Correlation & .051 & .091 & -.115 & .046 & $.254^{*}$ & -.134 & 1 \\
\hline & Sig. (2-tailed) & .637 & .398 & .282 & .665 & .016 & .210 & \\
\hline & $\mathrm{N}$ & 89 & 89 & 89 & 89 & 89 & 89 & 89 \\
\hline
\end{tabular}

**. Correlation is significant at the 0.01 level (2-tailed).

*. Correlation is significant at the 0.05 level (2-tailed).

\section{Discussion}

Oral manifestations were present in 88 out of 89 patients with CKD, who were included in this study. This represents the prevalence of $99.4 \%$. This is consistent with studies done from different geographical regions of the world, where the reported prevalence varies from $95 \%$ to $100 \%^{(13,}$ 5). Oral manifestations are usually due to malnutrition, neglect of oral health, restriction of fluids, immunosuppression due to CKD or drugs and the effects of drugs and ureamic toxins on the oral tissues ${ }^{(14)}$.Oral signs in CKD are decrease in salivary flow rate, xerostomia, oral candidiasis, periodontitis, high urea concentration in saliva, ammonia-like smell, stomatitis, pale gingivae, drug-induced gingival hyperplasia, oral bleeding, loss of lamina dura, maxillary and mandibular radiolucent lesions, abnormal bone remodelling after extraction, enamel hypoplasia, delayed tooth eruption pattern, low caries prevalence, dental erosion, sensitivity to percussion and mastication, tooth mobility and malocclusion ${ }^{(5)}$

In this study, the occurrence of dry mouth (xerostomia) was present in $19 \%$ of patients. The reasons postulated for the dry mouth include reduced salivary secretion due to fluid restriction advised in CKD, glandular involvement (atrophy of minor salivary glands), persistent inflammation, side effects of medication (mainly antihypertensives and diuretics), and mouth breathing. Similar to our study lower frequency of dry mouth was reported in $31 \%$ of patients by a study carried out by Uday Kumar et al from India and Murali et al who reported xerostomia in only $23 \%$ patients $^{(15,16)}$. Other studies showed a much higher prevalence of $56 \%$ and $91 \%{ }^{(17,5)}$. The higher values reported in other studies may reflect the underlying etiology of the kidney disease. The 
risk factors for CKD like diabetes mellitus, amyloidosis and autoimmune disease not only cause renal disease but also produce changes in the salivary gland ${ }^{(18)}$. In patients with CKD who have xerostomia, it predisposes the patient to dental caries, inflammation of the gingiva, oral candidiasis and sialadenitis ${ }^{(19)}$.

In this study we have performed periodontal assessment, using the CPITN index.. The periodontal examination included probing pocket depth, clinical attachment loss, bleeding on probing and presence of plaque. The Severity of chronic periodontitis is based on the amount of clinical attachment loss (CAL) and is graded as mild (1-2 mm CAL), moderate (3-4 mm CAL) or severe (> $5 \mathrm{~mm} \mathrm{CAL}$ )

In our study, the prevalence of periodontitis was 99.4\%. While severe chronic periodontitis was significantly more frequent among patients with grade $5 \mathrm{CKD}$. There are only a very few studies available previously, that evaluated the extent and severity of periodontal diseases in CKD patients; therefore, it is very difficult to compare our results with others. But the exacerbation of periodontal disease, as the disease progresses from early to late stage has been reported by other investigators. A study published by Borawski et al, based on the Community Periodontal Index of Treatment Needs and described a higher prevalence of severe periodontitis among predialysis, CAPD and HD patients compared with healthy individuals ${ }^{(7)}$. In another study published by Thorman et al. showed that predialysis and HD patients had significantly more clinical attachment loss than healthy subjects $^{(20)}$.

In our study, dental caries was found in $34 \%$ of patients. There is no consensus between authors whether dental caries are more common in patients with CKD. In a study published by A. Kaushik et al. there is an increased prevalence of dental caries in CKD and the reported prevalence is $45 \%{ }^{(21)}$. In yet another study published by Bayraktar $\mathrm{G}$ et al. showed higher prevalence of dental caries among CKD patients compared to healthy individuals ${ }^{(22)}$. Uremia, elevated $\mathrm{pH}$, decreased salivary flow rate, poor oral hygiene and an increase in the number of cariogenic Streptococcus mutans can cause high level of decayed tooth among these patients ${ }^{(23)}$. This is in contradiction to a study published by Sobrado Marinho JS et al. the number of decayed teeth was lower in the patients with CKD than in the controls (24). This is attributed to the antibacterial effect of increased $\mathrm{pH}$ as a result of urea hydrolization by saliva, which has a protective function against caries. And in a study published by Bots et al. there is no significant difference in decayed tooth among CKD patients $^{(25)}$.

In the present study, oral candidiasis was found to be present in $30 \%$ of patients. This is accordance with the study published by Royne and colleagues where the prevalence of candidal infections in $37 \%$ of CKD patients ${ }^{(26)}$. Oral candidiasis was more prevalent in CKD cases and may be due to immune suppression from malnutrition, restricted diets, anemia, stress, and immunosuppressive drugs. ${ }^{i}$ But in another study published by Gavalda and co-workers the reported prevalence of oral thrush is $5.7 \%{ }^{(27)}$. This variation may be due to the genetic variation, environmental factors and awareness of oral health among CKD patients.

Our study has a few limitations. First we have taken only a small group of individuals who had CKD. There is no controls to assess the impact of confounding factors like sex, socio economic status, smoking, previous dental visits, diabetes mellitus on the oral manifestations. An appropriate case control study should be established to eliminate the confounding factors. And finally, only the physical measurement of periodontal disease was made and it is not correlated with the effect of biochemical markers like Serum calcium, serum phosphorus, serum iPTH, and serum hsCRP on periodontitis.

However, large number of samples will help us in better understanding the interrelationship between oral pathological manifestations and kidney disease. 


\section{Conclusion}

Oral signs and symptoms are very common in patients with Chronic Kidney Disease. It is very clear that patients with chronic kidney disease are more likely to have periodontal disease. Though the literature on Indian population in this regard is very scarce, one study has shown higher prevalence of periodontal disease in CKD patients. The results showed an inverse relationship between GFR and periodontal disease. There is also an increased prevalence of xerostomia, decay, oral candidiasis in our patients. Recognition of the oral manifestations CKD is important, since they may be indicators of the presence of the disease. They also help in assessing the extent of CKD and the prognosis.

\section{References}

1. Andrew S. Levy, Kai-Uwe ECKARDT, Yusuke tsukamoto. Definition and classification of chronic kidney disease: A position statement from Kidney Disease: Improving global outcome (KDIGO) Kidney international, vol 67 (2005) PP 2089-2100

2. Ajay K Singh, Youssef MK Farrag, Bharati V Mittal Epidemiology and risk factors of chronic kidney disease in India from the SEEK (Screening and Early Evaluation of Kidney Disease) study BMC Nephrology 2013 14:114

3. Helen I McDonald, Sara L Thomas, Dorothea Nitsch Chronic kidney disease as a risk factor for acute community-acquired infections in high-income countries: a systematic review BMJ Open 2014;4:e004100

4. Cleber M Souza, Ana Paula R Braosia, Sônia M Luczyszyn,Rafaela W. Casagrande, Roberto Pecoits-Filho,Miguel C Riella, Sérgio A Ignácio, Paula C Trevilattoa Oral health in Brazilian patients with chronic renal disease Rev Med Chile. 2008 Jun;136(6):741-6
5. Patil, S., Khandelwal, S., Doni, B., Rahman, F. and Kaswan, S. Oral manifestations in chronic renal failure patients attending two hospitals in north Karnatka, India. OHDM, 2012; 11(3): 100-106

6. Loesche WJ, Grossman NS. Periodontal disease as a specific, albeit chronic, infection: diagnosis and treatment. Clin Microbiol Rev. 2001;14:727-52

7. Borawski J, Borawska WM, Stokowska W, Mys'liwiec M. The periodontal status of pre-dialysis chronic kidney disease and maintenance dialysis patients. Nephrol Dial Transplant 2007;22:457-464

8. Nadeem M, Stephen L, Schubert C, Davids MR. Association between periodontitis and systemic inflammation in patients with end-stage renal disease. SADJ 2009;64:470-473

9. NATIONAL KIDNEY FOUNDATION: K/DOQI Clinical Practice Guidelines for Chronic Kidney Disease: Evaluation, Classification and Stratification. Am J Kidney Dis 39 (Suppl 1):S1-S266, 2002

10. Cutress TW1, Ainamo J, Sardo-Infirri Jnt Dent $\mathrm{J}$. The community periodontal index of treatment needs (CPITN) procedure for population groups and individuals. Int Dent J. 1987 Dec;37(4):222-33.

11. Mahon CR, Lehman DC, Manuselis G. Textbook of diagnostic microbiology $3^{\text {rd }}$ edition. Philadelphia: Saunders; 2007

12. Guggenheimer J1, Moore PA, Rossie K Insulin-dependent diabetes mellitus and oral soft tissue pathologies: II. Prevalence and characteristics of Candida and Candidal lesions Oral Surg Oral Med Oral Pathol Oral Radiol Endod. 2000 May;89(5):570-6

13. Elijah O Oyetola , Foluso J Owotade Oral findings in chronic kidney disease: implications for management in developing countries Oyetola et al. BMC 
Oral Health (2015) 15:24 DOI 10.1186/s12903-015-0004-z

14. Proctor R, Kumar N, Stein A, Moles D, Porter S. Oral and dental aspect of chronic renal failure. J Dent Res. 2005;84:199-208

15. Udaykumar, P., Balasubramanian, S., Ramalingam, KS., Lakshmi, C., Srinivas, CR. and Mathew, A.,C. Cutaneous manifestations in patients with chronic renal failure on hemodialysis. IJDVL, 2006; 72(2): 119-25

16. Murali, P., Narasimhan, M., Periasamy, S. and Harikrishan, T.,C. A comparision of oral and dental manifestations in diabetic and non-diabetic uremic patients receiving hemodialysis. J Oral Maxillofac Pathol, 2012; 16(3): 374-79

17. Manley KJ1. J Ren Care. Saliva composition and upper gastrointestinal symptoms in chronic kidney disease 2014 Sep;40(3):172-9. doi: 10.1111/jorc.12062. Epub 2014 Mar 20.

18. Ulasi I, Chinwuba K. The enormity of chronic Renal Disease in Nigeria. The situation in a teaching Hospital in SouthEast Nigeria. Journal of Tropical Medicine volume 2010 Article ID 501957, 6 pages doi: 101155/2010/501957

19. Porter SR, Hegarty A, Scully C (2004). An update of the etiology and management of xerostomia. Oral Surg Oral Med Oral Patho Oral Radiol Endod 97: 28-46

20. Thorman R, Neovius $M$, Hylander B. Clinical findings in oral health during progression of chronic kidney disease to end-stage renal disease in a Swedish population. Scand J Urol Nephrol.2009;43(2):154-159

21. A. Kaushik, S. S. Reddy, ${ }^{1}$ L. Umesh, ${ }^{2}$ B. K. Y. Devi, ${ }^{1}$ N. Santana, ${ }^{1}$ and N. Rakesh ${ }^{1}$ Oral and salivary changes among renal patients undergoing hemodialysis: A cross-sectional study Indian J Nephrol. 2013 Mar-Apr; 23(2): 125-129

22. Bayraktar G, Kurtulus I, Duraduryan A, et al. Dental and periodontal findings in hemodialysis patients. Oral Dis 2007; 13:393-397

23. Gavaldá C, Bagán J, Scully C, Silvestre F, Milián M, Jiménez Y. Renal hemodialysis patients: Oral, salivary, dental and periodontal findings in 105 adult cases. Oral Dis 1999;5:299-302

24. Sobrado Marinho JS ${ }^{1}$, Tomás Carmona I, Loureiro A, Limeres Posse J, García Caballero L, Diz Dios P Oral health status in patients with moderate-severe and terminal renal failure Med Oral Patol Oral Cir Bucal. 2007 Aug 1;12(4):E305-10

25. Bots CP, Brand HS, Poorterman JH, et al. Oral and salivary changes in patients with end stage renal disease (ESRD): a two year follow-up study. $\mathrm{Br}$ Dent J2007;202:E3.

26. Royne T, Martin N, Britta H. Prevalence and early detection of fungi infection; A cross sectional controlled study in a group of Swedish end - stage renal disease patients. Scand J Urol Nephrol. 2009;43:325-30

27. Gavalda C, Bagan J, Scully C, Silvester F, Milian M, Jimenez Y. Renal hemodialysis patient; oral, salivary, dental and periodontal findings in 105 adult cases. Oral Dis. 1999;5:299-302. 